DOI: $10.1002 /$ adma.201603732

Article type: Communication

\title{
Shape-persistent Graphite Replica of Metal Wires
}

By Martin Pfeffermann, Michael Wuttke, Xinliang Feng, Akimitsu Narita, and Klaus Müllen*

M. Pfeffermann, M. Wuttke, Dr. A. Narita, Prof. Dr. K. Müllen

Max Planck Institute for Polymer Research,

Ackermannweg 10, 55128 Mainz (Germany)

E-mail: muellen@mpip-mainz.mpg.de

Prof. Dr. X. Feng,

Center for Advancing Electronics Dresden \& Department of Chemistry and Food Chemistry, Technische Universität Dresden,

Mommsenstraße 4, 01062 Dresden (Germany)

Keywords: Carbon, Conducting materials, Materials science, Templated synthesis

Graphite is an important carbon allotrope and an outstanding material in a variety of applications, including construction parts like screws and bolts, coatings, and electronics. ${ }^{[1-2]}$ The fabrication of different components from graphite is typically performed by cutting shapes from larger blocks. This method, however, unavoidably fabricates excessive waste and is only applicable for the production of large structures. Small ones in the micrometer range, such as micrometer-thin hollow carbon tubes, which offer opportunities in gas separation technology, catalysis, and water treatment, ${ }^{[3-5]}$ cannot be prepared with this process and require the use of bottom-up approaches. Procedures to obtain micrometer-thin graphite tubes so far include chemical vapor deposition, ${ }^{[6-10]}$ spinning, ${ }^{[5,11]}$ and the pyrolysis of cotton, ${ }^{[12-13]}$ charcoal, ${ }^{[14]}$ or polymers. ${ }^{[3-4,}{ }^{15-22]}$ These techniques, nevertheless, feature several disadvantages. For example, CVD-protocols generate graphite materials, referred to as multilayer graphenes, which coat the surface of an applied template. The resulting tubular structures, however, collapse upon removal of the templates and therefore fail to maintain their shapes. ${ }^{[6,9]}$ In contrast, the tubes obtained from pyrolyzing polymers were reported to be shape-persistent. This implies that the tubes sustained their macroscopic appearance without a template or other support, and without collapsing or bending. Nevertheless, all reported 
Submitted to

methods lack the possibility to customize the form of these tubes. Furthermore, these processes require high temperatures of $>2000{ }^{\circ} \mathrm{C}$ to yield a high quality of graphite. The quality of graphite material is essentially determined by the regularity of its layered framework of $\mathrm{sp}^{2}$-hybridized carbon atoms, showing an in-plane hexagonal arrangement, in combination with the crystallite sizes. ${ }^{[23-27]}$ The disadvantages of available techniques generate a pressing need for alternative and more versatile methods towards the formation of shape-persistent hollow carbon tubes. Here we report a facile one-step procedure for a template-assisted fabrication of hollow carbon tubes, using organic halides as carbon source (Fig. 1). The tubes are obtained as hollow replica of the metal template, maintain their shape, and do not collapse upon template removal. Further, additional etching steps are avoided as the template is removed during pyrolysis through the formation of metal halides, which are sublimed in-situ. Although pyrolyzed at relatively low temperatures up to $1000{ }^{\circ} \mathrm{C}$, our tubes feature a high quality of graphite, and a high conductivity of $1.31 \pm 0.05 \cdot 10^{6} \mathrm{~S} \cdot \mathrm{m}^{-1}$. With our approach, graphite microtubes with arbitrary shapes can be fabricated by using different templates.

In a typical experiment for producing hollow carbon tubes, $0.5-3 \mathrm{~cm}$ long iron wires with diameters of $130 \mu \mathrm{m}$ and $25 \mu \mathrm{m}$ as templates were placed in an evacuated quartz ampoule together with $100 \mathrm{mg}$ and $50 \mathrm{mg}$ of dichloromethane (DCM) as precursor, respectively. The ampoule was sealed and heated to $1000^{\circ} \mathrm{C}$ for $5 \mathrm{~h}$. After cooling to room temperature, the ampoule revealed black structures in the shapes and sizes of the provided metal wires (Fig. 1). Investigations of their cross-sections by scanning electron microscopy (SEM) at different positions confirmed the presence of hollow tubes. Providing $50 \mathrm{mg}$ and $100 \mathrm{mg}$ DCM together with wires of $25 \mu \mathrm{m}$ and $130 \mu \mathrm{m}$ in diameter gave hollow tubes with wall thicknesses of $1 \mu \mathrm{m}$ (Fig. 2a) and $23 \mu \mathrm{m}$ (Fig. 2b,c), respectively. The inner diameters complied with the ones of the templates. Tubes were broken apart and analyzed, corroborating 


\section{ADVANCED}

Submited to MATERIALS

the complete absence of the template wires (Fig. 2b). Magnifying the inner side of broken pieces (Fig. 2c) revealed a surface texture, which matches the template's surface (Fig. S2, supporting information). These results, showing the high similarity in shape, diameter, and morphology of the inner surface, demonstrate that the black structures are replica of the used templates.

To elucidate the reason for the absence of the template wire, a tube was investigated by energy-dispersive X-ray spectroscopy (EDX) by measuring a representative area on its surface (Fig. S3, supporting information) instead of the whole tube to avoid influences from the adhesive carbon pad. The EDX measurement revealed, apart from a high amount of carbon with 96 atom $\%$, the presence of oxygen with $1.8 \%$, iron with $0.5 \%$, and chloride with 1.1\% (Fig. S4, supporting information). Elemental mapping showed the uniform distribution of carbon, oxygen, chloride, and iron over the available surface (Fig. S3c-g, supporting information). Wet-chemical analyses with potassium hexacyanoferrate(II) and potassium hexacyanoferrate(III), giving evidence for the presence of both iron(II) and iron(III) species inside the tube material, in combination with the EDX results prove an in-situ reaction of the metal wire. Additionally, elemental distributions at cross-sections of the tube wall reveal continuously increasing iron as well as chloride contents from the inner side to $2 / 3$ through the wall thickness. After this point, both amounts of iron and chloride remain constant. (Fig. S5, supporting information). This behavior indicated that the applied wire first acted as a template, then reacted, and was finally removed by sublimation, whereas the stagnating area indicates the complete sublimation and thus a constant amount of iron and chloride in the gas phase.

The obtained tubes were subsequently analyzed by Raman spectroscopy. Criteria to judge the quality of graphite include the position and width of the G-band as well as the intensity ratios of the D-band to G-band $\left(I_{\mathrm{D}} / I_{\mathrm{G}}\right)$ and of the $2 \mathrm{D}$-band to G-band $\left(I_{2 \mathrm{D}} / I_{\mathrm{G}}\right)$, whereby low $I_{\mathrm{D}} / I_{\mathrm{G}}$ and 
high $I_{2 \mathrm{D}} / I_{\mathrm{G}}$ values support a high quality. ${ }^{[20,28-30]}$ The combination of DCM and iron gave a low $I_{\mathrm{D}} / I_{\mathrm{G}}$ ratio of 0.12 and a high $I_{2 \mathrm{D}} / I_{\mathrm{G}}$ ratio of 0.47 (Fig. $2 \mathrm{~d}$, see supporting information for optimization procedure). Contrary to the decreasing quality of template-assisted graphene formations upon incrementally increasing the numbers of layers,${ }^{[31-32]}$ our hollow carbon tubes feature a high quality of graphite even though possessing a relatively high thickness up to $23 \mu \mathrm{m}$.

The key role of an organic halide together with a metal wire was confirmed with two control experiments. First, using benzene with iron in the fabrication process only produced a carbon coating around the wire without its removal. Second, hexachlorobenzene (HCB) was pyrolyzed without a metal wire. High $I_{\mathrm{D}} / I_{\mathrm{G}}$ values were observed in the Raman spectra of both control experiments, indicating the formation of amorphous carbon and suggesting a low degree of graphitization without the combination of metal and organic halide (Fig. S6, supporting information). Silicon dioxide and silicon were also tested as templates, which furnished high $I_{\mathrm{D}} / I_{\mathrm{G}}$ values and broad $2 \mathrm{D}$ regions without any etching effect (Fig. S7 right, supporting information). Hollow carbon tubes were also generated using different metals, e.g. copper and gold, and different organic halides, namely chloroform $\left(\mathrm{CHCl}_{3}\right)$, perchlorocoronene (PCP), hexachlorobenzene (HCB), or hexabromobenzene (HBB). Indeed, the Raman spectra of all produced tube structures showed typical G- and D- peaks, and in some cases 2D peaks. ${ }^{[29]}$ Both copper and gold with DCM as precursor revealed good qualities of graphite, however, with more defects and smaller $I_{2 \mathrm{D}} / I_{\mathrm{G}}$ ratios (Fig. S7 right, blue, orange, and green, supporting information). The differences in graphite quality are presumably the result of different reactivities of the respective metal halides. $\mathrm{CHCl}_{3}, \mathrm{PCP}$, and HBB led to high $I_{\mathrm{D}} / I_{\mathrm{G}}$ values and broad $2 \mathrm{D}$ regions (Fig. S7 left, pink, black, and turquois, supporting information). $\mathrm{HCB}$ gave tubes with an $I_{\mathrm{D}} / I_{\mathrm{G}}$ value comparable to that with $\mathrm{DCM}$, yet with a lower $I_{2 \mathrm{D}} / I_{\mathrm{G}}$ ratio (Fig. S7 left, red, supporting information). The essential influence 
of the precursor structure on the formation of graphite material, suggests the following conclusions: 1) Chloride in contrast to bromide enhances the quality of graphite, representing the higher catalytic activity of $\mathrm{FeCl}_{3}$ compared to $\mathrm{FeBr}_{3} \cdot{ }^{[33-35]}$ 2) Providing aromaticity in the precursor does not enhance the quality. 3) Smaller molecules result in a higher regularity, which we ascribe to an increase in molecular mobility. 4) Raising the carbon/chloride ratio decreases the quality, presumably due to a reaction of excessive chloride with carbon material, resulting in more defective graphite.

We propose the following mechanisms during pyrolysis: 1) At lower temperatures of the heating procedure, the precursors are uniformly distributed in the ampoule by sublimation. 2) Upon further heating, the active metal surface catalyzes the dissociation of precursor to build an initial carbon coating on the template. Remaining halogens react with the metal to form metal halides, which promote the formation of graphite. 3) Higher temperatures cause a sublimation of metal halides to the vapor phase, allowing for a high quality of the produced micrometer-thick graphite and intercalating between the graphitic layers. 4) Eventually, heating to $1000^{\circ} \mathrm{C}$ finalizes the graphitization.

Considering the graphite hollow tubes as shape-persistent replica of the respective metal template, we changed the form of applied metal wires to obtain graphite materials with different structures. We thus fabricated hollow graphite architectures with knot, solenoid, mesh, toroid, and Y-shapes from corresponding metal templates as shown in Fig. 3. The knot displayed the general possibility to produce bent and/or twisted hollow carbon tubes. The solenoid (Fig. 3b) featured 33 turns, expressed a width of $1 \mathrm{~mm}$ and the hollow tube an inner diameter of $130 \mu \mathrm{m}$. The mesh (Fig. 3c) was produced with large dimensions of $5 \times 0.5 \mathrm{~cm}^{2}$. Its SEM picture revealed the woven structure and the hollow character of the tubes at the breaking points as depicted in Fig. S9b (supporting information). To demonstrate the possibility of connecting tubes, we fabricated a toroid (Fig. 3d, a magnified image of the 
junction is visible in Fig. S9a), featuring a diameter of $3.2 \mathrm{~mm}$, and a Y-shape, which connects three hollow tubes (Fig. 3e), both with inner diameters of $130 \mu \mathrm{m}$ (preparation of templates is given in the supporting information).

The electrical resistance of a linear hollow carbon tube with an inner diameter of $130 \mu \mathrm{m}$ and a wall thickness of $23 \mu \mathrm{m}$ (produced from DCM and iron, contacted with micromanipulator and measured with a four-wire resistance measurement configuration at incremental distances) featured an Ohmic behavior. Linear fitting of the measured resistances plotted against the wire length revealed a high conductivity of the hollow carbon tubes of $1.31 \pm 0.05 \cdot 10^{6} \mathrm{~S} \cdot \mathrm{m}^{-1}$ (Fig. S10, supporting information). This conductivity is almost one magnitude higher than the one reported for pristine graphite with $1.41 \pm 0.01 \cdot 10^{5} \mathrm{~S} \cdot \mathrm{m}^{-1} \cdot{ }^{[36]}$ As the conductivity of graphite was reported to essentially depend on the intercalation of impurities in the crystal lattice, ${ }^{[37]}$ we ascribe the obtained high conductivity to the inclusion of metal ions inside the material.

In summary, we introduced a one-step procedure for a facile fabrication of shape-persistent hollow carbon tubes with control over the wall thickness, inner diameter and shape of the tubes. The template was removed during the fabrication process, providing replica of metal wires. The in-situ formed metal halides were found to improve the graphite quality. Future research will focus on the use of the tubes in devices, exploiting their excellent conductivity and possibility to form arbitrary shapes. The carbon tube with a solenoid shape might have desirable electromagnetic properties and the carbon mesh could be used as electrode material in electrolysis. Further opportunities could result from the possible superconductive properties of graphite materials in conjunction with shape control of the objects. ${ }^{[38-42]}$

\section{Supporting Information}

Supporting Information is available online from the Wiley Online Library or from the author. 


\section{Acknowledgements}

Submitted to

Financial support by the ERC grant 2DMATER and the EC under the Graphene Flagship (grant number CNECT-ICT-604391) are gratefully acknowledged. The authors thank Gunnar Glasser for his kind help in SEM measurements. The authors apprechiate helpful discussions and support by Dr. Renhao Dong and Dr. Markus Mezger. ((Supporting Information is available online from Wiley InterScience or from the author)).

Received: ((will be filled in by the editorial staff))

Revised: ((will be filled in by the editorial staff)) Published online: ((will be filled in by the editorial staff))

[1] Y. Wei, Z. Sun, Curr. Opin. Colloid Interface Sci. 2015, 20, 311-321.

[2] P. Yu, S. E. Lowe, G. P. Simon, Y. L. Zhong, Curr. Opin. Colloid Interface Sci. 2015, 20,329-338.

[3] M. Speiser, S. Henzler, U. Hageroth, A. Renfftlen, A. Müller, D. Schawaller, B. Sandig, M. R. Buchmeiser, Carbon 2013, 63, 554-561.

[4] U. K. Fatema, C. Tomizawa, M. Harada, Y. Gotoh, Carbon 2011, 49, 2158-2161.

[5] Y. Zhao, C. Jiang, C. Hu, Z. Dong, J. Xue, Y. Meng, N. Zheng, P. Chen, L. Qu, ACS Nano 2013, 7, 2406-2412.

[6] T. Chen, L. Dai, Angew. Chem. Int. Ed. 2015, 54, 14947-14950.

[7] R. Wang, Y. Hao, Z. Wang, H. Gong, J. T. L. Thong, Nano Lett. 2010, 10, 4844-4850.

[8] X. Li, P. Sun, L. Fan, M. Zhu, K. Wang, M. Zhong, J. Wei, D. Wu, Y. Cheng, H. Zhu, Scientific Reports 2012, 2, 395.

[9] D. Mendoza, World J. Nano Sci. Eng. 2012, 2, 53-57.

[10] M. S. Dresselhaus, G. Dresselhaus, K. Sugihara, I. L. Spain, H. A. Goldberg, in Graphite Fibers and Filaments, Vol. 5, (Ed.: M. Cardona), Springer-Verlag Berlin Heidelberg, 1988.

[11] Y. Liu, H. G. Chae, Y. H. Choi, S. Kumar, J. Mater. Sci. 2015, 50, 3614-3621.

[12] B. Wang, R. Karthikeyan, X.-Y. Lu, J. Xuan, M. K. H. Leung, Ind. Eng. Chem. Res. 2013, 52, 18251-18261.

[13] S. Wang, Z. Ren, J. Li, Y. Ren, L. Zhao, J. Yu, RSC Adv. 2014, 4, 31300-31307.

[14] J. M. Skowroński, K. Knofczyński, Y. Yamada, Solid State Ionics 2003, 157, 133-138.

[15] L. Y. Jiang, T.-S. Chung, R. Rajagopalan, Carbon 2007, 45, 166-172.

[16] J. Qiu, T. Qiu, Carbon 2015, 81, 20-28.

[17] W. Xie, H. Cheng, Z. Chu, Z. Chen, C. Long, Ceram. Int. 2011, 37, 1947-1951.

[18] Z.-G. Shi, T. Zhang, L.-Y. Xu, Y.-Q. Feng, Microporous and Mesoporous Mater. 2008, 116, 698-700.

[19] H. Zengin, D. W. Smith, J. Mater. Sci. 2007, 42, 4344-4349.

[20] U. K. Fatema, F. Okino, Y. Gotoh, J. Mater. Sci. 2013, 49, 1049-1057.

[21] J. Sun, X. Wang, C. Wang, Q. Wang, J. Appl. Polym. Sci. 2006, 99, 2565-2569.

[22] M.-C. Yang, D.-G. Yu, Text. Res. J. 1996, 66, 115-121.

[23] M. A. Pimenta, G. Dresselhaus, M. S. Dresselhaus, L. G. Cancado, A. Jorio, R. Saito, Phys. Chem. Chem. Phys. 2007, 9, 1276-1290.

[24] A. C. Ferrari, J. Robertson, Phys. Rev. B 2000, 61, 14095-14107.

[25] I. Rannou, V. Bayot, M. Lelaurain, Carbon 1994, 32, 833-843.

[26] H. Wilhelm, M. Lelaurain, E. McRae, B. Humbert, J. Appl. Phys. 1998, 84, 6552-6558.

[27] F. Tuinstra, J. L. Koenig, J. Chem. Phys. 1970, 53, 1126-1130.

[28] A. C. Ferrari, Solid State Commun. 2007, 143, 47-57. 


\section{Submitted to

[29] L. M. Malard, M. A. Pimenta, G. Dresselhaus, M. S. Dresselhaus, Physics Reports 2009, 473, 51-87.

[30] P. Lespade, R. Al-Jishi, M. S. Dresselhaus, Carbon 1982, 20, 427-431.

[31] M. Bayle, N. Reckinger, J.-R. Huntzinger, A. Felten, A. Bakaraki, P. Landois, J.-F. Colomer, L. Henrard, A.-A. Zahab, J.-L. Sauvajol, M. Paillet, Phys. Status Solidi B 2015, 252, 2375-2379.

[32] H. J. Park, J. Meyer, S. Roth, V. Skakalova, Carbon 2010, 48, 1088-1094.

[33] G. A. Olah, S. Kobayashi, M. Tashiro, J. Am. Chem. Soc. 1972, 94, 7448-7461.

[34] B. Plietker, in Iron Catalysis: Fundamentals and Applications, Vol. 33, (Eds.: M. Beller, J. M. Brown, P. H. Dixneuf, A. Fürstner, L. S. Hegedus, P. Hofmann, T. Ikariya, L. A. Oro, M. Reetz, Q.-L. Zhou), Springer-Verlag Berlin Heidelberg, 2011.

[35] S. Cenini, F. Ragaini, in Catalytic Reductive Carbonylation of Organic Nitro Compounds, Vol. 20, (Eds.: R. Ugo, B. R. James), Springer-Science+Business Media, B.V., 1997.

[36] X. Luo, R. Chugh, B. C. Biller, Y. M. Hoi, D. D. L. Chung, J. Electron. Mater. 2002, 31, 535-544.

[37] H. Zabel, S. A. Solin, in Graphite Intercalation Compounds. II. Transport and Electronic Properties, Vol. 18, Springer-Verlag Berlin Heidelberg, 1992.

[38] L. Grover, V. Yuriy, H. Kiar, Supercond. Sci. Technol. 2016, 29, 015015.

[39] J. Zhou, Q. Sun, Q. Wang, P. Jena, Phys. Rev. B 2015, 92, 064505.

[40] R. P. Smith, T. E. Weller, C. A. Howard, M. P. M. Dean, K. C. Rahnejat, S. S. Saxena, M. Ellerby, arXiv.org, e-Print Arch., Condens. Matter 2015, 1-22.

[41] R. D. Parks, Science 1964, 146, 1429-1435.

[42] H. Yamamoto, H. Nasu, H. Kameno, Physica C 2003, 392-396, Part 1, 759-763.

[43] M. Ballester, C. Molinet, J. Castañer, J. Am. Chem. Soc. 1960, 82, 4254-4258.

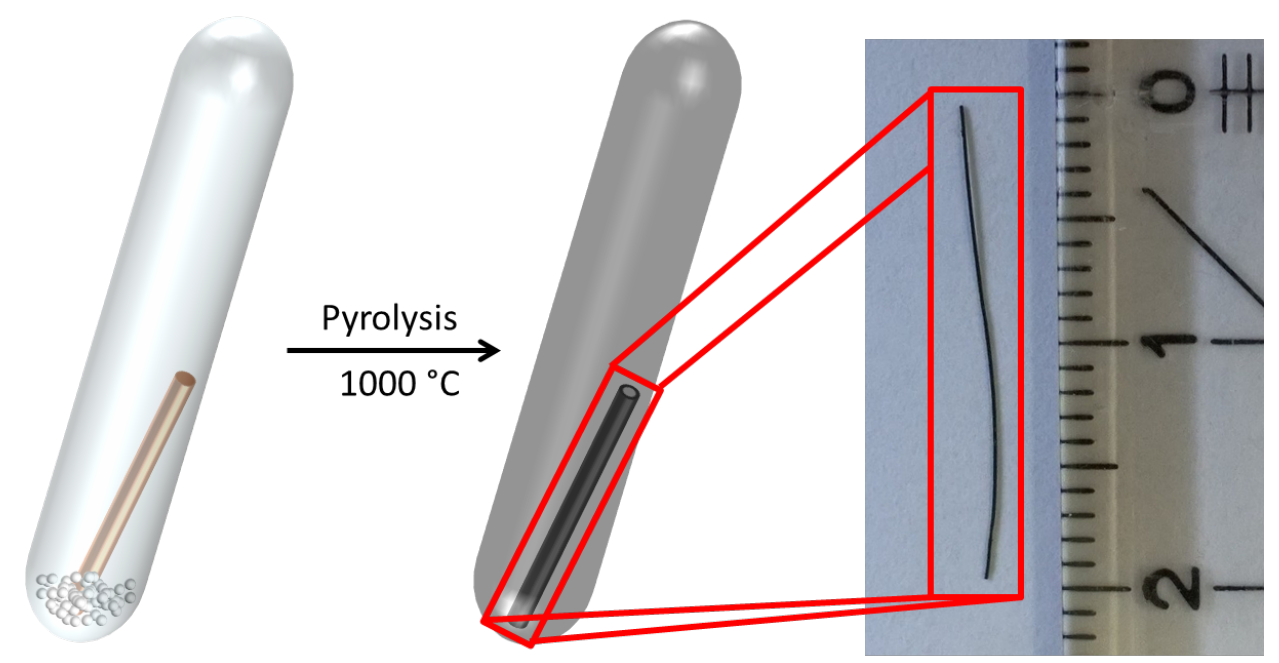

Figure 1. Schematic procedure for the preparation of hollow carbon tubes. The left side shows an evacuated ampoule, providing both a metal template and the precursor. The middle reveals the ampoule after pyrolysis. The right picture shows the obtained wire after pyrolysis. 

Submitted to HATERAS


Figure 2. SEM images of hollow carbon tubes. a) The tube features a wall thickness of $1 \mu \mathrm{m}$ and was produced from an iron wire with a diameter of $25 \mu \mathrm{m} . \mathrm{b}$ ) This tube was fabricated from an iron wire of $127 \mu \mathrm{m}$ in diameter and fractured to reveal its continuous hollowness. The wall thickness is $23 \mu \mathrm{m}$. c) The magnified area of the inner side of a broken piece reveals a surface texture, which matches the surface morphology of the template's surface (Figure S2). d) Raman spectrum of a hollow carbon tube, produced from DCM on an iron wire with a ratio of $\mathrm{Fe} / \mathrm{Cl}=1 / 3$.
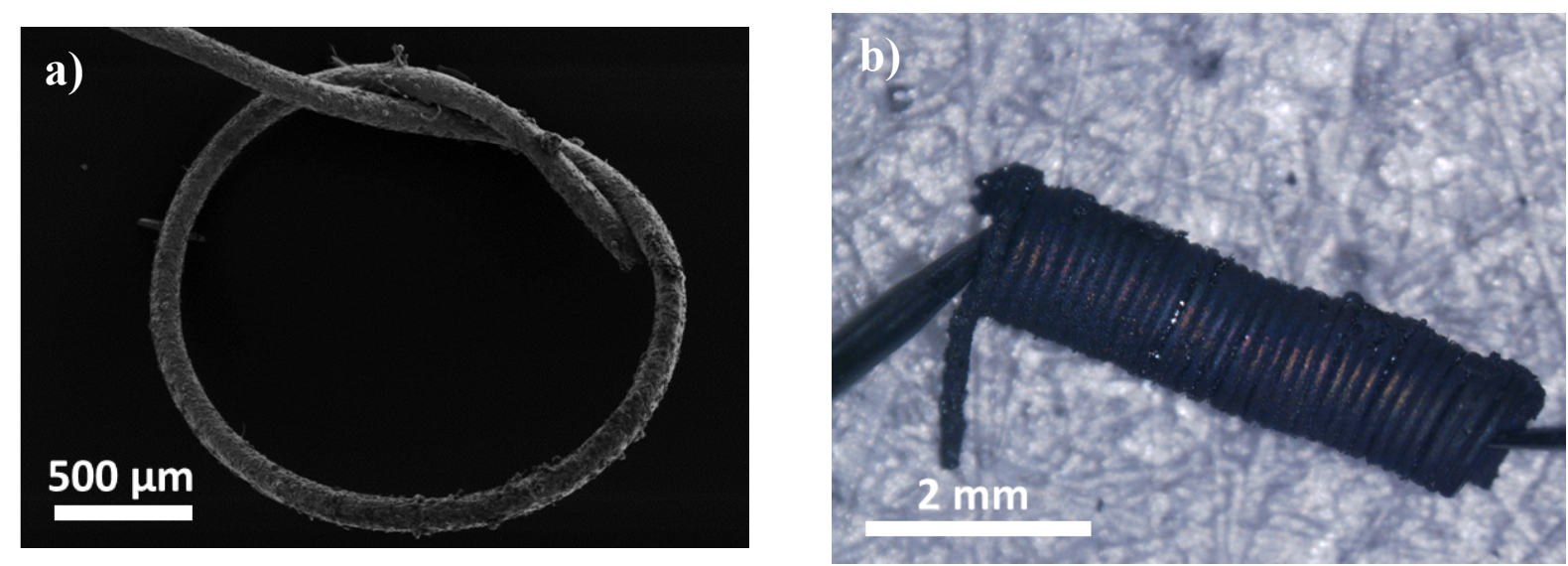


\section{Submitted to

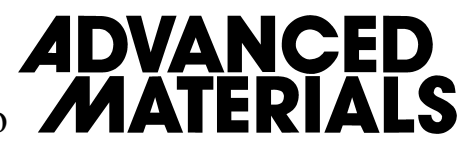

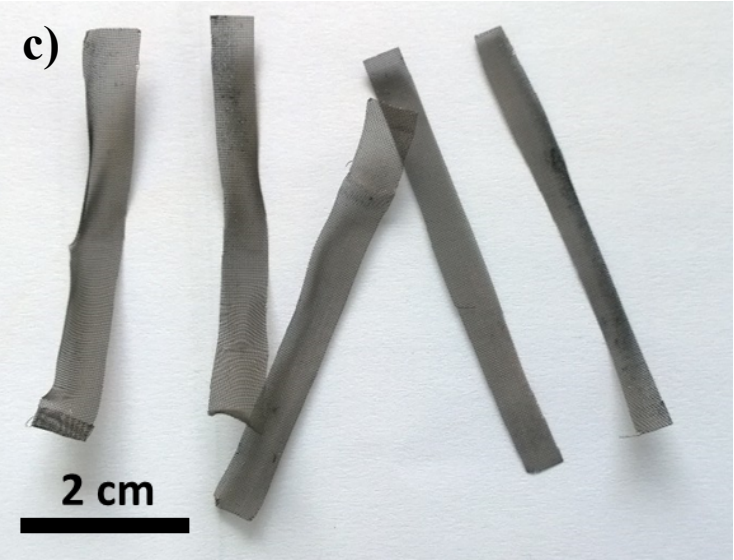
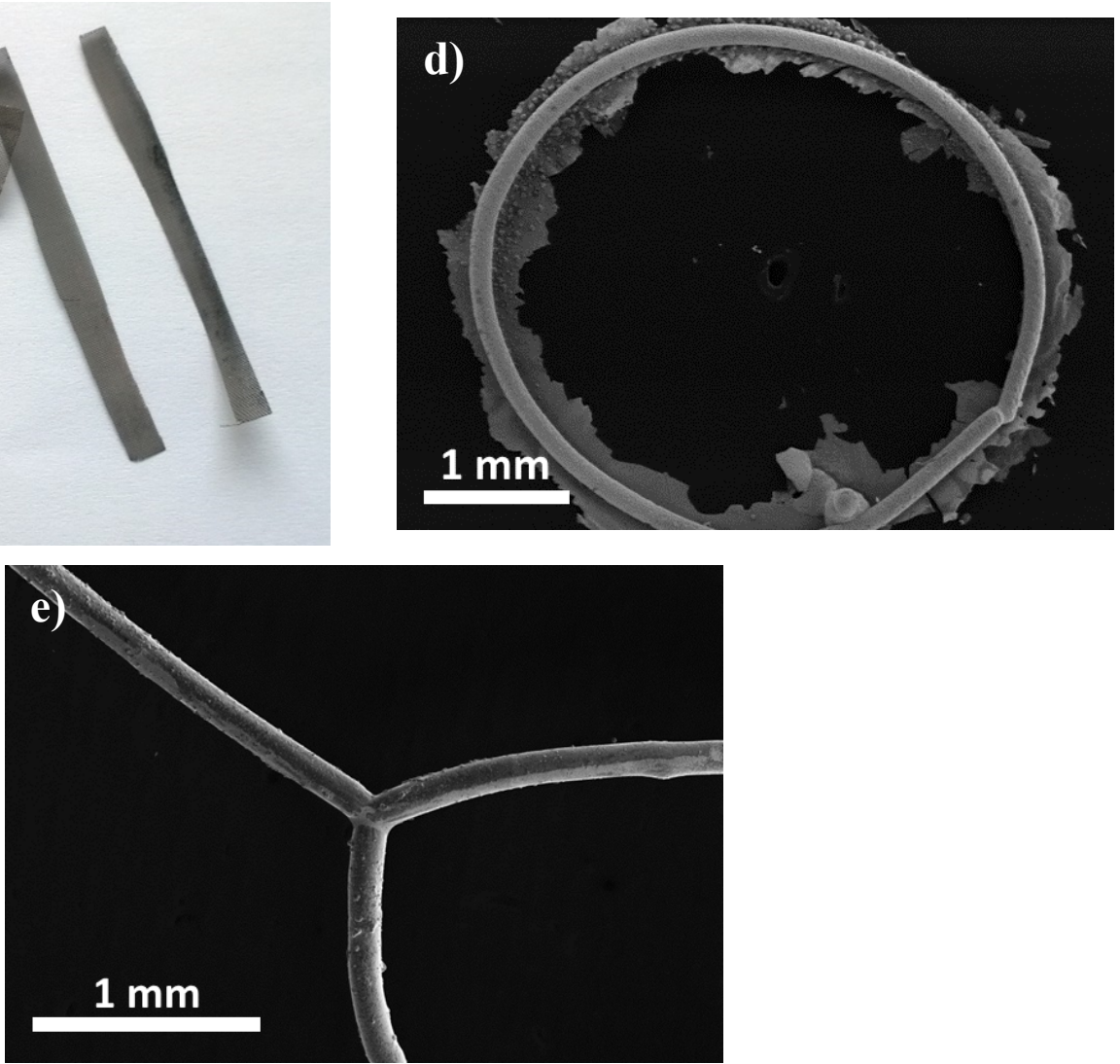

Figure 3. Different shapes of hollow carbon tubes. The figures show a) a SEM picture of a knot, b) an optical microscopy picture of a solenoid, c) a photograph of a mesh, d) a SEM picture of a toroid, and e) a SEM picture of a Y-shape. 
The table of contents entry

Submitted to

We report a facile one-step procedure for a template-assisted fabrication of hollow carbon tubes, using organic halides as carbon source. The tubes are shape-persistent and obtained as hollow replica of the metal template, and they do not collapse after removal of the template. Further, we avoid additional etching steps as the template is removed during pyrolysis through the formation of metal halides which are in-situ sublimed. Although pyrolyzed at relatively low temperatures up to $1000^{\circ} \mathrm{C}$, our tubes feature a high quality of graphite, and a high conductivity of $1.31 \pm 0.05 \cdot 10^{6} \mathrm{~S} \cdot \mathrm{m}^{-1}$. With our approach, graphite microtubes with arbitrary shapes can be fabricated by using different templates.

Keyword: Carbon, Conducting materials, Materials science, Templated synthesis.

Martin Pfeffermann, Michael Wuttke, Xinliang Feng, Akimitsu Narita, and Klaus Müllen* Shape-persistent Graphite Replica of Metal Wires

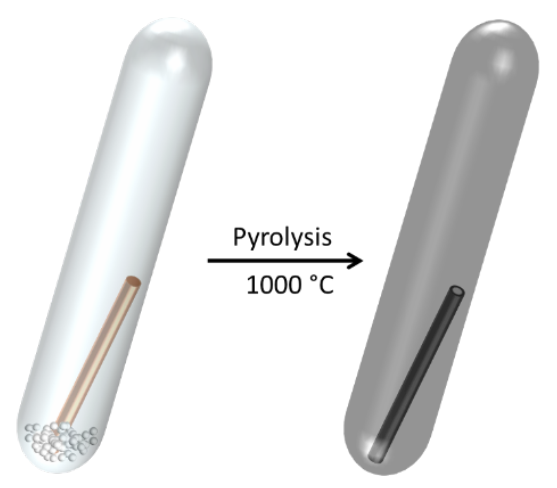


Copyright WILEY-VCH Verlag GmbH \& Co. KGaA, 69469 Weinheim, Germany, 2013.

\section{Supporting Information}

for $A d v$. Mater., DOI: 10.1002/adma.((please add manuscript number))

Shape-persistent Graphite Replica of Metal Wires

Martin Pfeffermann, Michael Wuttke, Xinliang Feng, Akimitsu Narita, and Klaus Müllen*

General information:

All commercially available chemicals and solvents were used as purchased without further purification.

Raman spectra were recorded at a laser wavelength of $488 \mathrm{~nm}$ and an intensity of $2 \mathrm{~mW}$ with a Bruker RFS 100/S instrument, equipped with a Senterra Dispersive Raman Microscope. The peak positions and intensities were obtained from fitting the peaks with Lorentz functions.

SEM measurements were performed on a LEO Gemini 1530 with samples taped with conductive carbon pads to aluminum stubs. EDX measurements were carried out on a Hitachi SU8000 device. Resistances were measured with a Keithley model 4200 semiconductor characterization system, whereas contacting was performed under a Nikon SMZ1000 microscope.

Template preparation:

Applied templates were purchased as available at Alfa Aesar, Sigma Aldrich, or Goodfellow. All templates were cleaned by ultrasonication with aceton, toluene, and isopropanol prior to 
Submitted to

\section{ADNANCES
MAATERALIS}

providing them in an ampoule. Copper templates were additionally treated with an aqueous

$2 \mathrm{M}$ hydrochloric acid solution for the removal of copper oxides on the surface.

For preparing the copper toroids, a copper wire with a diameter of $127 \mu \mathrm{m}$ was cut into pieces of $0.6 \mathrm{~cm}$. The wire pieces were bent in such a way that the cutting edges touch each other. The bent pieces were placed into a custom made graphite template (Figure S1, left, supporting information), which was positioned into an oven. The oven was permanently flushed with a nitrogen flow of $1300 \mathrm{~cm}^{3} \cdot \mathrm{min}^{-1}$ to prevent oxidation of the copper. After heating the oven to $1100{ }^{\circ} \mathrm{C}$ with a heating rate of $5{ }^{\circ} \mathrm{C} / \mathrm{min}$, the temperature was kept constant for $18 \mathrm{~min}$. Letting the oven cool to room temperature and removing the copper wires from the template resulted in the cutting edges being fused to a toroid in nine of ten cases.

The Y-pieces were prepared similar to the copper toroids, however with copper wire pieces of $0.4 \mathrm{~cm}$. Fusing the cutting edges in the oven was performed under similar conditions as with the toroids, however with a temperature plateau of $7 \mathrm{~min}$ at $1100{ }^{\circ} \mathrm{C}$ and an appropriate graphite template (Figure S1, right, supporting information).


Figure S1. Pictures of the graphite templates used for fusing the cutting edges (top) to obtain copper toroids (left), and copper Y-shapes (right), respectively. Below every template, an example of the respective copper shape is shown. 


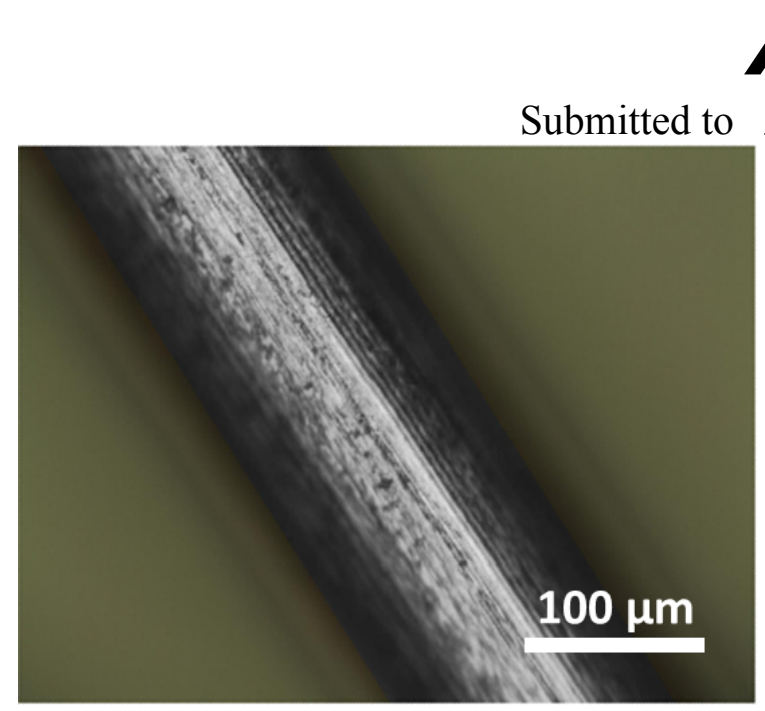

ADNANCES
MAATRRALIS

Figure S2. Optical microscopy picture of a bare iron wire. The surface clearly reflects the characteristic texture, as found inside the hollow tubes in Figure 2c. 
Elemental mapping of a hollow carbon tube:

\section{ADVANCED
MATERIALS Submitted to MATERIALS}
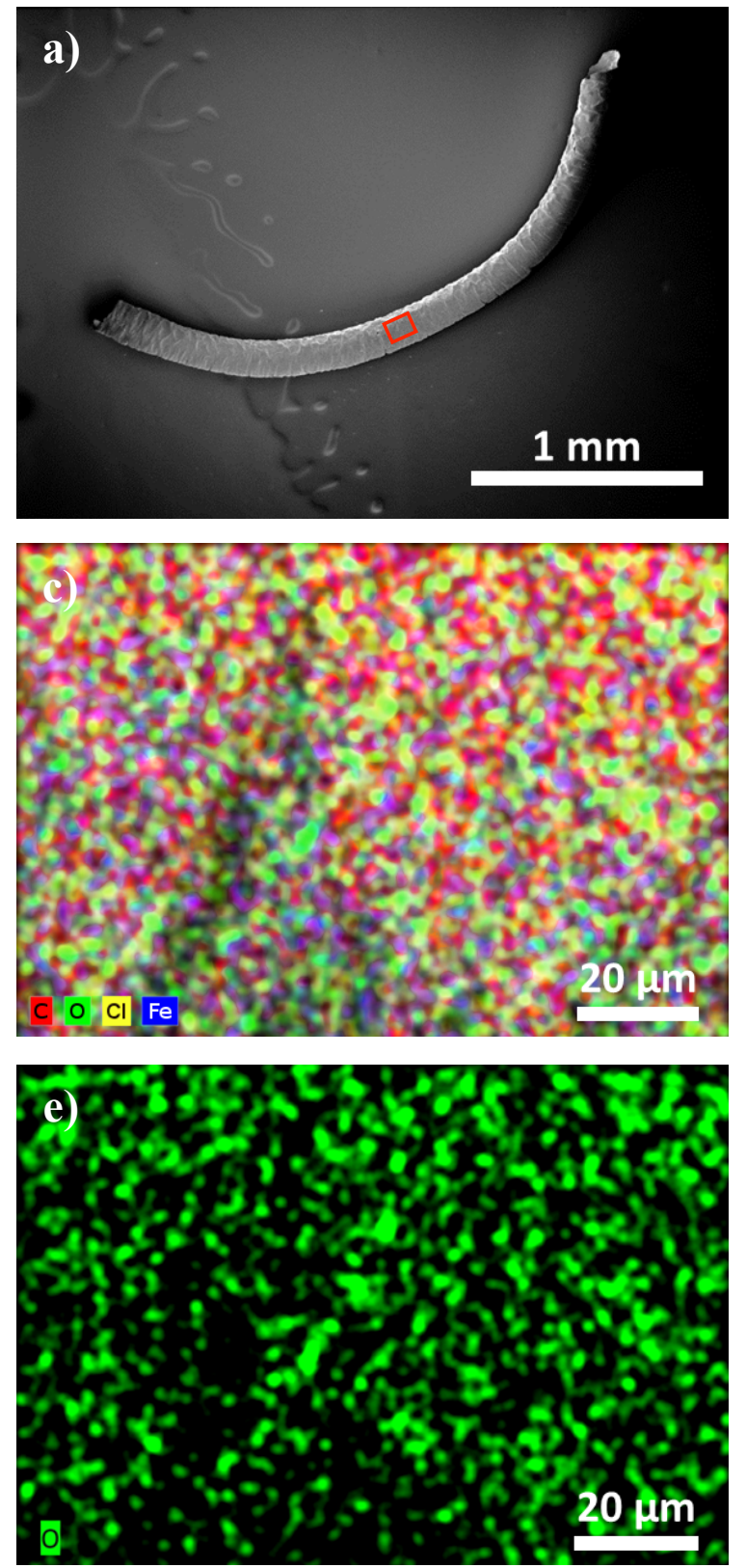



d)

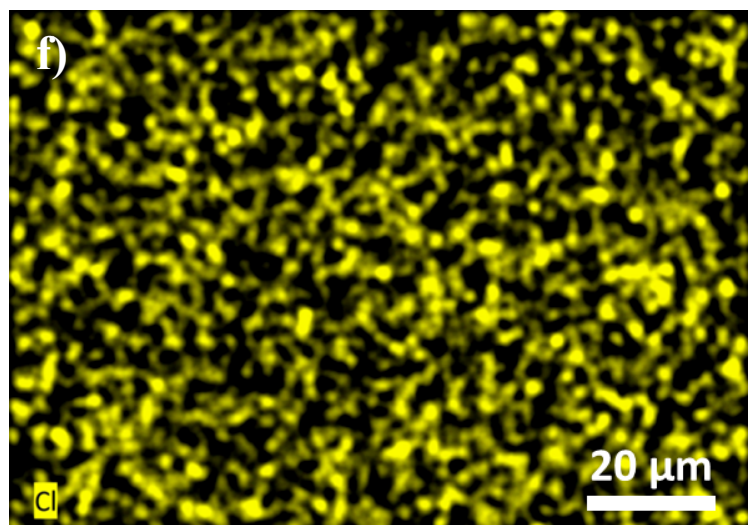

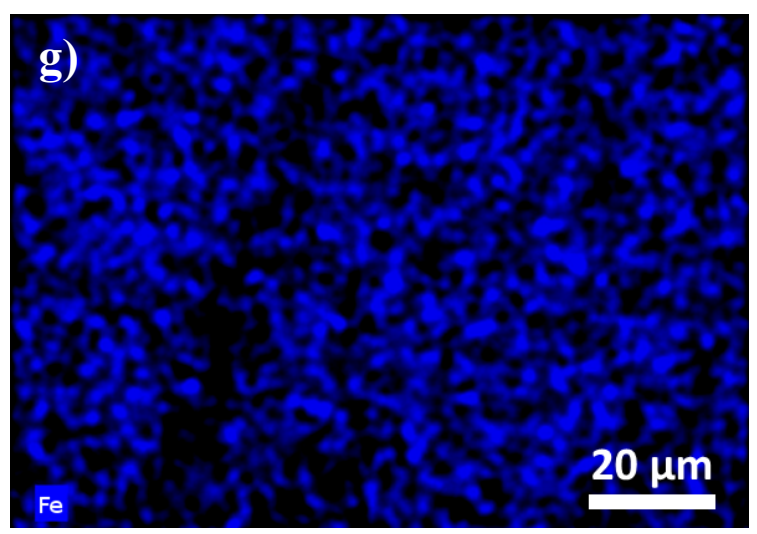

Figure S3. a) SEM image of the measured hollow carbon tube. The red frame highlights the area of higher magnification in b). b) SEM image of the area of the tube, used for elemental mapping. Small differences in contrast appear due to varying scattering angles of incident 


\section{Submitted to

electrons, induced by marginal undulations of the tube. These differences in intensity are also visible in the elemental mapping images of $\mathrm{c}-\mathrm{g}$ ). c) Elemental mapping of carbon, oxygen, chloride, and iron. d) Elemental mapping of carbon. e) Elemental mapping of oxygen. f) Elemental mapping of chloride. g) Elemental mapping of iron.

\section{EDX-measurement:}



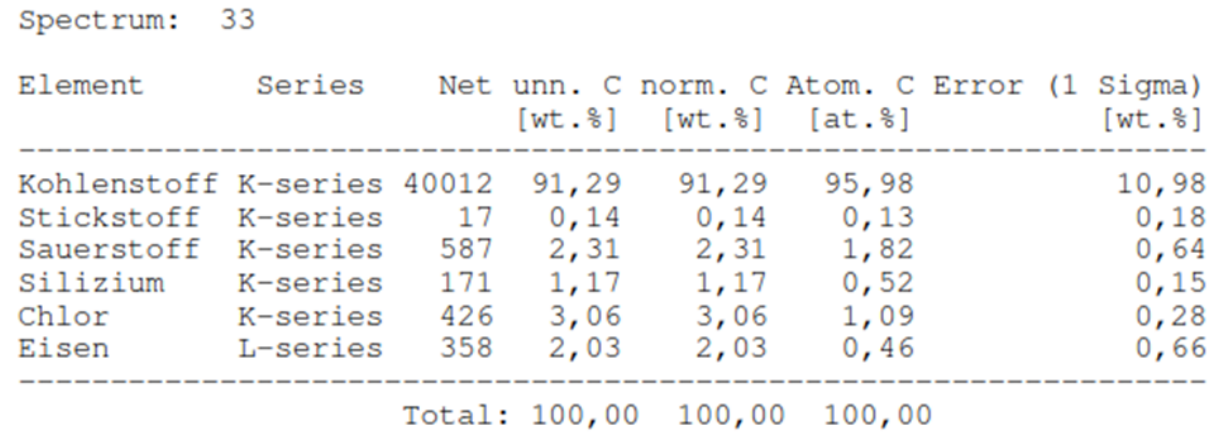

Figure S4. EDX data of the selected area of the hollow carbon tube in Figure S3b.

The measured traces of silicon were residues from the quartz ampoule. 
a)



b)



c)

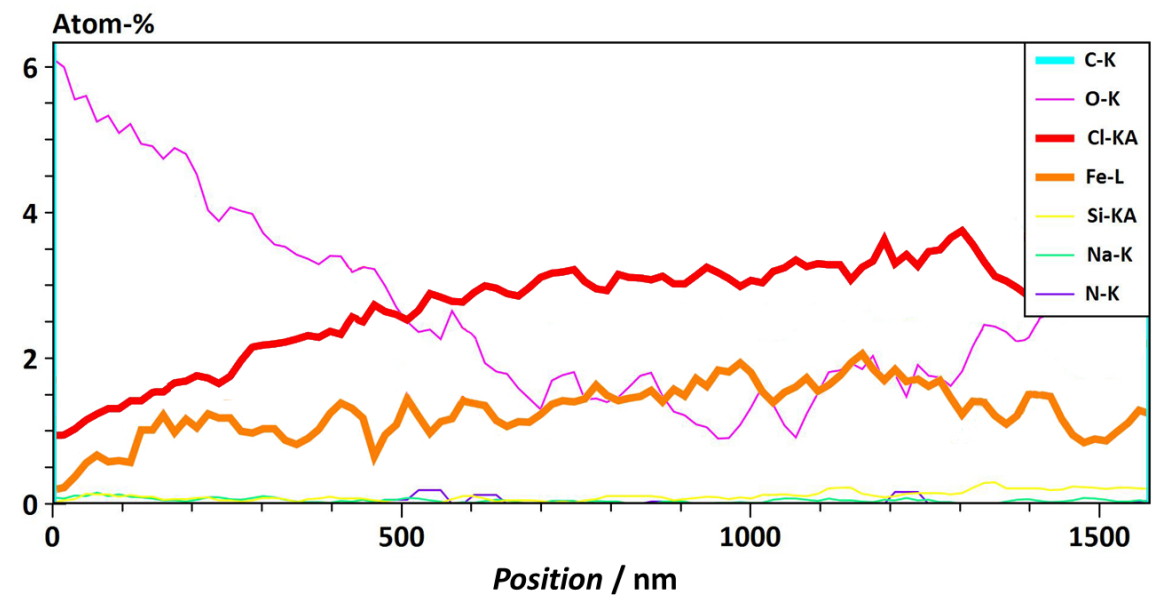

Figure S5. a) SEM image of the measured cross section of a hollow carbon tube wall. The left side represents the inner part of the tube, while the right side faces towards the outside. The blue line highlights the area of measurement. b) Plot of the atomic distribution in percent against the position of measurement on the blue line in a). The elements carbon (turquoise), oxygen (pink), chlorine (red), iron (orange), silicon (yellow), sodium (green), and nitrogen (purple) were plotted. The measured traces of silicon, sodium, and nitrogen are not significant for interpretation. c) Magnified plot of b) for the less represented elements. 
Raman spectra:

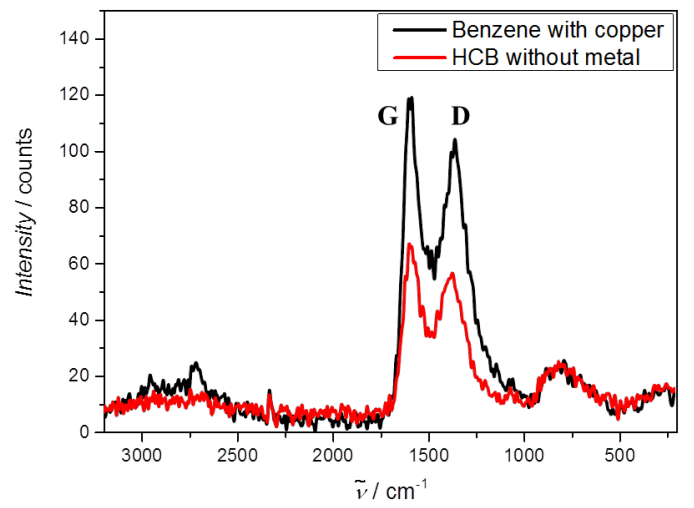

Figure S6. Raman spectra of the pyrolysis of benzene with copper (black), and of HCB without the presence of a metal (red).


Figure S7. Raman spectra of different precursors in combination with an iron wire template (left), and of the most promising precursor dichloromethane on different surfaces (right). Low qualities of graphite were obtained with $I_{\mathrm{D}} / I_{\mathrm{G}}$ of $1.41,1.21$, and 0.96 using perchlorocoronene (black), hexabromobenzene (turquois), and chloroform (pink) as precursors, respectively. This low quality is corroborated by the insignificant, broad 2D regions. Higher qualities were achievable providing hexachlorobenzene (red), and dichloromethane (green), giving $I_{\mathrm{D}} / I_{\mathrm{G}}$ ratios of 0.67 and $I_{2 \mathrm{D}} / I_{\mathrm{G}}$ ratios of 0.37 and 0.61 , respectively. Pyrolysis of DCM achieved an $I_{\mathrm{D}} / I_{\mathrm{G}}$ ratio of 0.88 on silicon dioxide, and $I_{\mathrm{D}} / I_{\mathrm{G}}$ and $I_{2 \mathrm{D}} / I_{\mathrm{G}}$ ratios of 0.97 and 0.35 on singlecrystalline silicon, 0.67 and 0.37 on copper, 0.21 and 0.51 on iron, and 0.69 and 0.46 on gold, respectively.

Optimization of the combination of iron and DCM:

With the combination of DCM and iron as the most promising precursor and template material, respectively, three different ratios, $\mathrm{Fe} / \mathrm{Cl}=1 / 3,1 / 2$, and $1 / 6$ were tested. A high ratio of chlorine atoms of $\mathrm{Fe} / \mathrm{Cl}=1 / 6$ (Figure $\mathrm{S} 8$, black, supporting information) resulted in a low graphite quality with $I_{\mathrm{D}} / I_{\mathrm{G}}$ and $I_{2 \mathrm{D}} / I_{\mathrm{G}}$ ratios of 1.03 and 0.28 , respectively. In contrast, a low 


\section{Submitted to \\ ADVANCED}

ratio of chlorine atoms to $\mathrm{Fe} / \mathrm{Cl}=1 / 2$ (Figure $\mathrm{S} 8$, red, supporting information) gave a low $I_{\mathrm{D}} / I_{\mathrm{G}}$ ratio of 0.20 and an $I_{2 \mathrm{D}} / I_{\mathrm{G}}$ ratio of 0.35 . However, in this case the iron template was not completely removed and therefore the tubes were not entirely hollow. $\mathrm{Fe} / \mathrm{Cl}=1 / 3$ revealed a high graphite quality with a low $I_{\mathrm{D}} / I_{\mathrm{G}}$ ratio of 0.12 and an $I_{2 \mathrm{D}} / I_{\mathrm{G}}$ ratio of 0.47 . The optimized spectrum reveals an intensive G-peak at $1580.3 \mathrm{~cm}^{-1}$, a small D-peak at $1362.6 \mathrm{~cm}^{-1}$, and a significant 2D-peak at $2732.5 \mathrm{~cm}^{-1}$

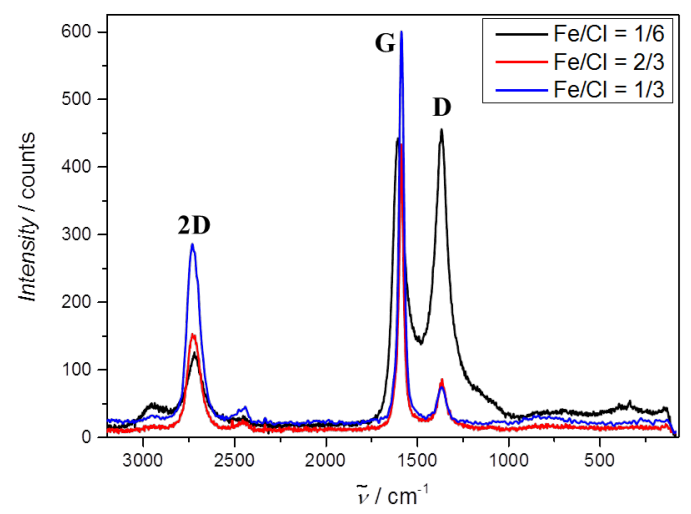

Figure S8. Raman spectra of the pyrolysis of DCM on an iron template with different iron to chloride ratios. The black spectrum shows a ratio of $\mathrm{Fe} / \mathrm{Cl}$ of $1 / 6$, the red spectrum of $1 / 2$, and the blue spectrum of $1 / 3$. The $I_{\mathrm{D}} / I_{\mathrm{G}}$ and $I_{2 \mathrm{D}} / I_{\mathrm{G}}$ ratios were obtained as 1.03 and 0.28 for the black spectrum, 0.20 and 0.35 for the red spectrum, 0.12 and 0.47 for the blue spectrum, respectively.

Additional SEM picures:
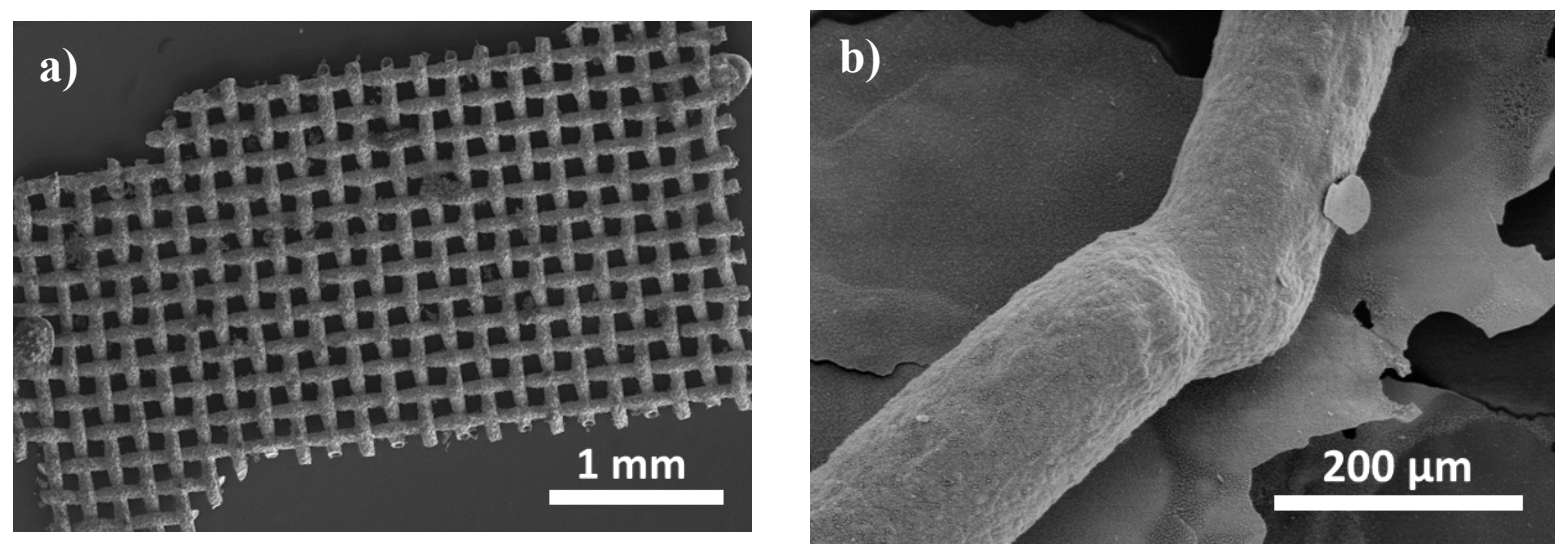

Figure S9. a) SEM image of a hollow graphite mesh. The hollowness is corroborated by the breaking point of the individual tubes. b) Magnified SEM image of the point juncture in the toroid. 
Conductivity measurement:

Submitted to

The resistance of a hollow carbon tube with an inner diameter of $127 \mu \mathrm{m}$ and a wall thickness of $23 \mu \mathrm{m}$ was measured. The wire was fixed on both ends with silver varnish to simplify contacting. Each end was contacted and a current of $2.5 \mathrm{~mA}$ was applied. Between the two contacts, two needles were positioned on the wire with incremental distances of $1 \mathrm{~mm}$. Between these needles, the voltages were measured and the respective resistances calculated by Ohm's law. Latter were plotted against the distances in Figure S10. Fitting the measurement with a linear fit revealed a resistance of the hollow tube of $1.56 \pm 0.07 \cdot 10^{-}$ ${ }^{1} \Omega \cdot \mathrm{mm}^{-1}$. Regarding a cross-section area of $5.00 \cdot 10^{-3} \mathrm{~mm}^{2}$, the resistivity $\rho_{\text {Tube }}$ of the tube was calculated using the equation

$$
\rho=\frac{R \cdot A}{l}
$$

with $R$ being the resistance, $A$ as cross-section area, and $l$ as length of the tube, to be $\rho_{\text {Tube }}=7.66 \pm 0.35 \cdot 10^{-7} \Omega \cdot \mathrm{m}$. The conductivity $\sigma_{\text {Tube }}$ as reciprocal value of the resistivity is therefore $\sigma_{\text {Tube }}=1.31 \pm 0.05 \cdot 10^{6} \mathrm{~S} \cdot \mathrm{m}^{-1}$.

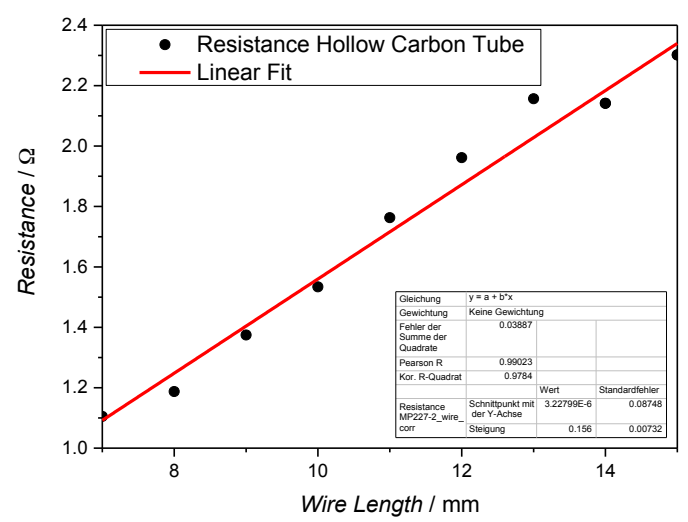

Figure S10. Resistance measurements of a hollow carbon tube at incremental distances. The linear fit reveals an inclination of $1.56 \pm 0.07 \cdot 10^{-1} \Omega \cdot \mathrm{mm}^{-1}$. 
Precursors:

Dichloromethane (DCM), chloroform, hexahlorobenzene, hexabromobenzene, and pentachloropyridine were commercially available and used without further purification. DCM and chloroform were frozen with liquid nitrogen prior to evacuation of the ampoule to prevent evaporation of the precursor.

Perchlorocoronene was synthesized by perchlorination under BMC conditions $^{[43]}$ of commercially available coronene. $750 \mathrm{mg}(5.63 \mathrm{mmol}) \mathrm{AlCl}_{3}$ were refluxed in $280 \mathrm{~mL}$ sulfuryl chloride. $3.00 \mathrm{~g}(9.99 \mathrm{mmol})$ coronene was dispersed with $1.50 \mathrm{~g}$ (11.1 mmol, $0.89 \mathrm{~mL}$ ) disulfur dichloride in $120 \mathrm{~mL}$ sulfuryl chloride and slowly added to the reaction. The mixture was refluxed for $30 \mathrm{~h}$ and $50 \mathrm{~mL}$ sulfuryl chloride were added for dissolving occurring precipitation. Subsequently, the solvent was evaporated and the residue poured into ethanol. The occurring precipitate was filtered and washed with ethanol, aqueous $1 \mathrm{M}$

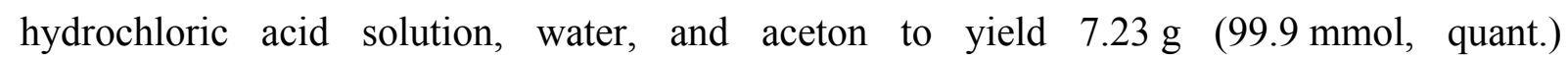
perchlorocoronene as yellow powder. ${ }^{13} \mathrm{C} \mathrm{NMR}\left(125.7 \mathrm{MHz}, \mathrm{C}_{2} \mathrm{D}_{2} \mathrm{Cl}_{4}, \delta\right): 132.8(\mathrm{C} 4), 126.4$ (C4), $121.1(\mathrm{C} 4)$; MALDI-MS (m/z): $713.2\left[\mathrm{M}^{+}\right]$, calc. $713.6\left[\mathrm{C}_{24} \mathrm{Cl}_{12}\right]$. 


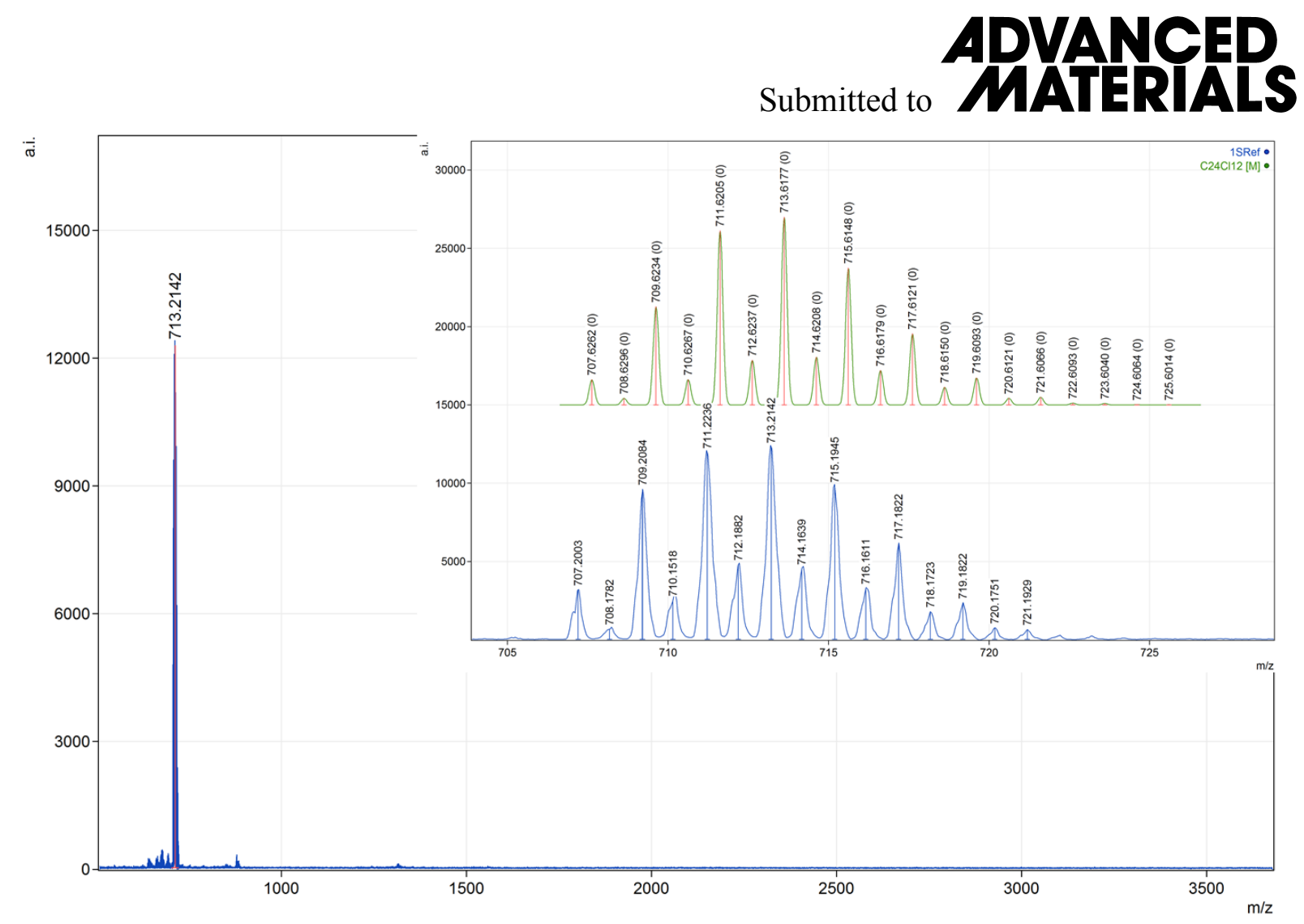

Figure S11. MALDI-MS spectrum of PCC. The inset reveals the measured isotope pattern (blue) compared to the calculated one for $\mathrm{C}_{24} \mathrm{Cl}_{12}$ (green). 